\title{
Mumpreneurs: más allá de la relación "Emprendimiento-maternidad"
}

\author{
Mumpreneurs: beyond the "Entrepreneurship-motherhood" relationship \\ Silvia C. Acosta-Velázquez ${ }^{a}$, Elba M. Pedraza-Amador ${ }^{b}$
}

\begin{abstract}
:
The role that culturally and socially is assigned to women as responsible for the home and family care has undergone transformations over time for two main reasons; the first one is based on circumstances that lead them to become head of the family, and the second attends to the desire for economic independence and professional development, which has allowed the creation of spaces for them in the workplace, where they carry out activities until little considered exclusive to the male gender.

However, it seems that the conditions of inequality, lack of opportunities for growth in the world of work and the female nature itself play against the aspirations of working women to reconcile both roles, since their life after motherhood often gives a turn that leads them to change their priorities and consider the dilemma of leaving their job to take care of the new member of the family or sacrificing their personal life for their professional development.

This search for a mother-worker balance creates a new dynamic and gives rise to a phenomenon that is gaining strength around the world, that of female mumpreneurs, which is addressed in this article with the purpose of discovering, based on an exhaustive review from the available literature, what lies beyond the maternity-entrepreneurship relationship and what advantages does the reconciliation of both roles mean for the female sector.
\end{abstract}

\section{Keywords:}

Mumpreneurs, female entrepreneurship, labor discrimination, gender inequality

\section{Resumen:}

El rol que cultural y socialmente se adjudica a las mujeres como responsables de la casa y el cuidado de la familia ha sufrido transformaciones al paso del tiempo por dos razones principales; la primera está en función de circunstancias que las llevan a convertirse en cabeza de familia, y la segunda atiende al deseo de independencia económica y desarrollo profesional, lo que ha permitido la creación de espacios para ellas en el ámbito laboral, en donde desempeñan actividades hasta poco consideradas ex clusivas del género masculino.

Sin embargo, pareciera que las condiciones de desigualdad, falta de oportunidades de crecimiento en el mundo laboral y la propia naturaleza femenina juegan en contra de las aspiraciones de las mujeres trabajadoras de conciliar ambos roles, pues es frecuente que su vida después de la maternidad dé un giro que las lleva a cambiar sus prioridades y plantearse la disyuntiva de abandonar su empleo para atender al nuevo integrante de la familia o sacrificar su vida personal en pro de su desarrollo profesional.

Esta búsqueda de equilibrio madre-trabajadora crea una nueva dinámica y da origen a un fenómeno que cada vez cobra mayor fuerza alrededor del mundo, el de las mumpreneurs, que se aborda en este artículo con el propósito de descubrir, a partir de una exhaustiva revisión de la literatura disponible, qué hay más allá de la relación maternidad-emprendimiento y qué ventajas supone para el sector femenino, la conciliación de ambos roles.

\section{Palabras Clave:}

Mumpreneurs, emprendimiento femenino, discriminación laboral, desigualdad de género

Autor de Correspondencia, Universidad Autónoma del Estado de Hidalgo, https://orcid.org/0000-0001-8972-7748, Email: silvia_acosta2066@uaeh.edu.mx

b Universidad Autónoma del Estado de Hidalgo, https://orcid.org/0000-0002-7182-2437, Email: elbam@uaeh.edu.mx 


\section{Introducción}

El emprendimiento femenino es una situación cotidiana en el mundo actual. Cada vez son más las mujeres que eligen fundar y dirigir su propio negocio ante la falta de oportunidades en un entorno laboral complejo, caracterizado por la sobrecarga de trabajo y la discriminación de género.

El principal argumento que esgrimen en apoyo a su decisión de emprender es la posibilidad de organizar su horario para realizar las tareas propias de la gestión familiar, y demostrar con ello que es posible combinar la maternidad con las actividades empresariales.

En este sentido, nótese que a pesar de las acciones que los gobiernos han tomado para erradicar la discriminación por género, la brecha existente en la participación laboral entre hombres y mujeres es una realidad, y pareciera que la inclusión de éstas en las actividades económicas y la remuneración percibida por su trabajo está en función del estado conyugal y el número de hijos (Vela, 2017), de tal suerte que las mujeres solteras o sin hijos tienen mayores posibilidades de ingresar al mercado de trabajo, aunque con remuneraciones inferiores a las percibidas por los hombres.

Lo anterior se fundamenta en información proveniente de diversos estudios realizados por la Organización Internacional del Trabajo (OIT), que refieren a la maternidad como una de las causas de discriminación laboral femenina, condicionante del acceso al empleo y en muchas ocasiones, motivo de la separación del mismo en función de la incompatibilidad de horarios para el cuidado de los hijos y permanencia en el mercado de trabajo (OIT, 2010), por lo que se le considera también como una de las principales dificultades para la generación o sostenimiento de ingresos provenientes de una relación laboral (OIT, 2016), evidenciando que la población femenina es uno de los grupos con menores oportunidades para el acceso a empleos bien remunerados en función de la matern idad y las licencias para cuidados que ello implica (Larraitz, Chaves, Carcedo, \& Sánchez, 2019).

Se observan así las graves violaciones a los derechos humanos y laborales de las mujeres, las conductas discriminatorias manifestadas en los horarios extremos, la segregación y/o despido por embarazo (COPRED, 2015) o por intentar hacer uso de los derechos sobre protección a la maternidad (Gamboa \& Valdés, 2008), condiciones que contravienen lo que establece la Ley Federal del trabajo (LFT) e impiden la conciliación de su maternidad con el trabajo.
Adicional a esto, el rol que cultural y socialmente se ha dado a la mujer a lo largo de la historia las confina a las tareas domésticas y de servicio y al cuidado de los hijos, y únicamente a partir del alistamiento de los hombres en las fuerzas armadas durante la primera guerra mundial, es que a sus labores en el hogar se suma su participación en la vida laboral, aunque con salarios menores y condiciones de trabajo infrahumanas, situación que subsiste en la actualidad.

En este contexto de desequilibrio en las responsabilidades del hogar, desigualdad laboral, escaso apoyo por parte de los empleadores, violación de derechos humanos y laborales, no hay lugar para la concertación maternidadempleo, por lo que en la búsqueda de desarrollo profesional y realización personal ejerciendo su maternidad, cada vez son más las mujeres que optan por el emprendimiento, encontrando en esa dinámica la oportunidad para desarrollar un negocio y atender una familia.

Ser madre, profesionista y tomar la decisión de emprender da lugar a un nuevo tipo de emprendimiento y a la generación de un término que engloba a madres jóvenes, con una nueva mentalidad, estudios de educación superior y experiencia laboral en mandos medios: "mumpreneur".

\section{El fenómeno "mumpreneur"}

El término "mumpreneur" es un sustantivo que de manera informal se usa en el Reino Unido para designar a "una madre que ha empezado su propio negocio" (Cambridge University Press, 2020), surgido a raíz de un concurso organizado por un programa televisivo para madres con ideas de negocios, a finales del año 2008.

Una variante de este sustantivo es la palabra "mompreneur" usado principalmente en Estados Unidos y Canadá para referir, también de manera informal, a las madres que combinan el emprendimiento con el cuidado de sus hijos (Collins English Dictionary, 2020).

El vocablo fue usado inicialmente en Francia, país con mayor tasa de nacimientos y presencia femenina en actividades empresariales, para describir a mujeres madres entre 25 y 45 años, que además de pugnar por la independencia económica y la libertad personal, buscan el equilibrio entre su vida laboral y familiar mediante el desarrollo de sus propios negocios, sin embargo, su verdadero origen proviene del movimiento pionero surgido en la década de los ochentas en Estados Unidos (Villaécija, 2011). 
Si bien las características de este fenómeno -referido indistintamente como mumpreneuro mompreneuren los diversos países- ameritan el desarrollo de un concepto aparte que lo distinga del emprendimiento en general, como sugieren Richomme-Huet \& Vial (2014), la literatura no aporta información significativa al respecto, refiriendo estudios aislados como el realizado en Australia por Nel, Maritz \& Thongpravati (2009), que se enfoca en analizar la integración de la maternidad y el emprendimiento y aborda, además del deseo de equilibrar el trabajo y la vida, otros factores que motivan a las mujeres a tomar la decisión de iniciar sus propios negocios.

Para estos autores, las mumpreneurs son las precursoras de una tendencia emergente con contribuciones importantes a las economías locales y mundial, a través de la creación de nuevas empresas que generan empleos, y más allá del incremento a sus ingresos y la conciliación vida-trabajo, buscan sentirse satisfechas consigo mismas, ganar respeto e igualar el desequilibrio de género.

En otros estudios, como el realizado en Italia en el año 2014 por el Instituto Nacional de Estadística (ISTAT por su sigla en italiano) se refiere la tasa de empleo femenina como la más baja de Europa, con una tendencia al descenso en función de su situación familiar y el número de hijos, de tal forma que a pesar de la experiencia acumulada y la preparación profesional, ante la falta de oportunidades en el ámbito laboral, eligen lanzarse a una aventura por cuenta propia como la única alternativa posible para conciliar la vida privada y laboral (Di Battista, 2107), situación que ha comenzado a convertirse en el referente de un nuevo estilo de vida, orientado a mejorar la calidad de vida familiar sin descuidar el empleo, a pesar de que el fenómeno no está muy extendido en este país.

En México, aunque el número de mujeres-madresemprendedoras se ha incrementado en fechas recientes, no debe perderse de vista que la presencia femenina al frente de negocios propios no es una situación nueva, pues a diferencia de las mujeres de otros países, una gran cantidad de mexicanas son el único respaldo económico de sus familias, por lo que tan sólo en el año 2011 existían alrededor de 16 millones de emprendedoras (Vargas, 2011) buscando equilibrar el papel de madres de familia y dueñas de negocios.

Por otra parte, al igual que en otros países alrededor del mundo, una gran cantidad de mumpreneurs mexicanas decidió independizarse después de sufrir discriminación en el trabajo al convertirse en madre, por lo que conviene analizar la relación que existe entre el mercado de trabajo y la maternidad, y los efectos que tiene para una mujer el tener hijos cuando se está sujeta a una relación laboral.

\section{Maternidad y Mercado Laboral}

No obstante que el ejercicio paralelo de la maternidad con actividades productivas pareciera no representar mayor dificultad para la mayoría de las mujeres alrededor del mundo, la situación se torna más compleja cuando éstas se encuentran sujetas a una relación laboral formal.

Relegadas tradicionalmente al espacio doméstico (Alcañiz, 2015), el acceso de las mujeres al mercado de trabajo generalmente se da a través de la informalidad, donde encuentran una alternativa para obtener ingresos a la par que permanecen al cuidado de los hijos y las labores del hogar (Bueno, 2009). Esta situación implica un doble trabajo debido a la ausencia de la figura masculina en la atención de la familia, y una carga de género con la cual muchas mujeres se incorporan al empleo remunerado en condiciones desfavorables, al margen de las regulaciones laborales.

La sobrecarga de trabajo, la desvalorización, la falta de oportunidades de ascenso, la invisibilización del trabajo femenino son comunes en las distintas sociedades, aunado a la percepción de su falta de capacidad para asumir sus actividades profesionales ante la presión que suponen las demandas de su centro de trabajo y las familiares y personales (Alcañiz, 2015), lo que ha propiciado que en la actualidad, las mujeres retrasen la maternidad, la eviten o incluso decidan tener menos hijos de los que consideraban dentro de sus expectativas de vida.

Ser madre y trabajadora no parece una tarea fácil. El rol productivo y reproductivo a que hacen referencia Brunet\& Santamaría (2016) las tipifica como trabajadoras de segunda clase, cuya participación se encuentra sujeta a la necesidad de incrementar el presupuesto familiar, o a circunstancias que la colocan como cabeza de familia ante la ausencia de una figura masculina, lo cual implica su inserción al ámbito laboral en ramas o actividades poco valoradas, con bajos salarios y escasas o nulas prestaciones.

Pareciera entonces que la maternidad y las actividades profesionales son incompatibles (Fernández, 2019); las madres, sobre todo aquellas jóvenes, son excluidas o expulsadas del mercado laboral bajo la perspectiva de los altos costos que supone la maternidad a las empresas. Adicionalmente, Todaro, Godoy \& Abramo (2002) refieren que los empresarios estiman un bajo grado de compromiso por parte de las madres trabajadoras, además de las restricciones para desempeñarse durante jornadas largas o realizar viajes y el incremento en el ausentismo, derivado de la fuerte presencia que requieren 
tener en sus hogares y el establecimiento de su vida familiar como prioridad.

Si bien tales consideraciones no justifican la exclusión de las madres del mercado de trabajo, es un hecho que en él están presentes diversas manifestaciones de discriminación asociadas a la maternidad, permitidas y admitidas en los diferentes países, lo cual obliga a este segmento de la sociedad a buscar alternativas de conciliación vida-trabajo, encontrando en los proyectos emprendedores, la posibilidad de ejercer libremente su maternidad, desarrollar sus proyectos personales y profesionales, y al mismo tiempo, percibir ingresos.

\section{Estrategias de conciliación vida-trabajo}

Las conclusiones de estudios sobre conciliación de la vida familiar y el trabajo, sin importar el género, apuntan a una mayor satisfacción con el empleo, compromiso con la empresa y mayores beneficios, sin embargo, la realidad sugiere que existe un desequilibrio importante, en tanto ambas actividades requieren presencia fuerte $y$ frecuentemente se otorga más tiempo a las actividades laborales que a las personales y familiares. En el contexto femenino, este desequilibrio alcanza las proporciones de un conflicto y en función de ello, no son pocas las mujeres que abandonan sus empleos o bien optan por un trabajo a tiempo parcial (Rodríguez \& Fernández, 2010) dependiendo del número de hijos que tengan.

En cuanto al trabajo que desempeñan, no obstante que el número de mujeres que se incorporan al ámbito empresarial a nivel mundial tiene una tendencia al crecimiento, las tasas de participación femenina y su acceso a puestos directivos se sitúan por debajo de los estándares masculinos, esto, sugieren las autoras que aseveran dicho planteamiento, se debe a que sus responsabilidades familiares son percibidas como una amenaza a su rendimiento laboral, además de que al buscar un empleo, las mujeres exploran, en primera instancia, alternativas compatibles con sus obligaciones como madres de familia.

Este fenómeno que refiere las preferencias por un tipo de trabajo determinado y la decisión de emplearse en él para estar en condiciones de asumir el cuidado de sus hijos y sus responsabilidades domésticas a la par que generan ingresos, es conocido como "suelo pegajoso" (OXFAM México, 2020) y constituye uno de los factores de mayor incidencia en la feminización del trabajo, es decir, en empleos a tiempo parcial y menores salarios (Gómez Bueno, 2001) que las mujeres realizan, generalmente en sector terciario o de servicios, para facilitar la conciliación con su vida familiar.
En relación al conflicto entre trabajo femenino y familia, Campillo (2010) refiere el término "conciliación" al abordar la bú squeda del equilibrio entre el trabajo remunerado y el entorno doméstico, sugiriendo que las medidas que prevalecen en el mercado laboral son fuente de nuevas desigualdades manifestadas a través de dos tipos de presencias: una de tiempo completo para el sexo masculino y las jornadas a tiempo parcial, con condiciones de desventaja, para las mujeres, en su intento por compaginar ambos roles.

Alcañiz (2015) por su parte, refiere una conciliación desigual y expone diversas estrategias, utilizadas por las mujeres madres de familia para lograr el cumplimiento de ambos roles, a saber: a) conciliación de supervivencia, b) conciliación organizada y c) conciliación mercantilizada [8].

La primera de ellas alude a reducción de jornadas y horarios flexibles adecuados a los intereses familiares de mujeres que generalmente no cuentan con estudios superiores y realizan trabajos manuales, en tanto que la conciliación organizada incluye estrategias de apoyo de la red familiar o pago de ayuda externa para completar sus jornadas de trabajo, utilizadas por mujeres en puestos de trabajo semiprofesionales o de oficina, y por último, la conciliación mercantilizada propia de mujeres madres de familia en empleos cualificados, con estudios superiorese interés en desarrollar una carrera profesional, que eligen negociar para mantener su posición laboral y en función de ello, no asumir en exclusiva la responsabilidad del hogar sino compartirla con su pareja.

Evidentemente, como señala la autora, la conciliación es desigual en tanto que las estrategias señaladas no pueden ser aplicadas de manera irrestricta, pues son elegidas por las mujeres en función de su posición laboral, su nivel socio-económico y su formación académica, dejando en claro que la conciliación vida-trabajo es una tarea pendiente que requiere la participación de las empresas y los gobiernos y la creación de políticas públicas de apoyo a las responsabilidades familiares, sin embargo, como ya se ha señalado, prevalece la decisión femenina de emprender un negocio propio para lograr el equilibrio entre ambas actividades, lo que da lugar a una nueva dinámica de emprendimiento.

\section{"Mum-entrepreneurship" como estrategia de conciliación}

El mum-entrepreneurship es una elección de vida que requiere coraje, entusiasmo y dedicación, no únicamente para resolver o contribuir a las necesidades económicas 
de la familia sino como una forma de alcanzar el reconocimiento social y desarrollo personal.

Cabe señalar que las mujeres tienen rasgos diversos en el comportamiento y decisiones frente al emprendimiento, por ejemplo, de acuerdo con estudios realizados por Andersson (2018) en países europeos, la decisión de las mujeres por autoemplearse a menudo está relacionada con la familia más que por razones económicas. Dicho estudio reveló que las mujeres que inician un emprendimiento previo a la maternidad pueden dedicar más tiempo al negocio lo que a menudo se convierte en un factor para la decisión de retrasar la maternidad o no decidir no ejercerla; en cambio quienes se convierten en emprendedoras cuando ya tienen hijos son más eficientes, ya que se relaciona con la capacidad de desarrollo de nuevas habilidades derivadas del cuidado del niño y la organización de la familia en general.

Por su parte Brunet y Pizzi (2017) refieren que las madres emprendedoras con hijos en los primeros años de vida, inician sus proyectos de negocio durante los primeros años, principalmente porque el autoempleo ofrece flexibilidad en el tiempo y cantidad de trabajo con la posibilidad eventual de trabajar en el hogar. En el estudio que realizaron en España, los autores comentan que para las madres emprendedoras "conciliadoras", la familia es el pilar alrededor del cual organizan sus tareas de cuidado y afecto hacia los hijos. Esto supone un cambio en el rol ya que logran conciliar tanto el trabajo productivo como el reproductivo que sigue estando muy presente a pesar de la inclusión de la mujer en el ámbito económico.

Es así que el emprendimiento como proyecto de vida para muchas mujeres, buscará flexibilidad para una mejor conciliación trabajo-familia, tal como lo plantean Genin, Michaudville, \& Cisneros, (2017) quienes definen que existen factores de necesidad (push factors) y factores de atracción (pull factors) hacia el emprendimiento, los primeros a menudo se observan como condiciones que impulsan a las mujeres a emprender por necesidad $u$ obligación y los segundos representan las razones por las cuales se motivan a iniciar un proyecto de emprendimiento por interés o identificación de una oportunidad interesante de negocio y el deseo de tomarla para ganar más dinero.

Por ello, a pesar de los retos que supone lograr esa conciliación entre el trabajo y la familia, además las mumpreneurs buscan el bien mayor e incluso pueden encontrar oportunidades de negocio debido a la insatisfacción o frustración con los productos y servicios que encuentran en el mercado principalmente en aquellos relacionados con la maternidad, los niños o la familia. Ejemplo de lo anterior se observa en la diversidad de inventos a lo largo de la historia moderna que han sido desarrollados pormujeres, tal el caso de Marion Donovan, arquitecta y madre de familia estadounidense, quien es considerada la precursora del pañal desechable, ya que en 1951 le fue otorgada la patente por haber creado una cubierta impermeable para pañales cuyo invento su rgió de la frustrante y repetitiva tarea de cambiar los pañales de tela sucios de sus hijos (Smithsonian Magazine, 2019).

Esto hace que las madres emprendedoras además de conciliar la relación trabajo-familia, también pueden lograr el reconocimiento y satisfacción personal, el incremento de sus ingresos, mejora de su posición en cuanto a independencia e igualdad de género (Nel, Maritz, \& Thongprovati, 2010).

\section{Conclusiones}

Las dificultades que enfrentan las madres de familia para conciliar las necesidades familiares con las largas y rígidas jornadas de oficina son únicamente uno de los tantos desafíos que implica su incorporación al mundo de trabajo. A ello se suma la falta de apoyos para el cuidado de sus hijos, insatisfacción en el trabajo, falta de flexibilidad laboral, elevados índices de desempleo y discriminación, restricciones para participación o crecimiento en las empresas, falta de reconocimiento a sus derechos laborales, invisibilización de su trabajo, y la creencia ancestral de que su realización se encuentra en la maternidad, que al pasar del tiempo, se han convertido en fuerzas de atracción para lograr el cumplimiento de sus expectativas profesionales de manera independiente.

Si bien la maternidad y el desequilibrio en la relación familia-trabajo influyen directamente en las decisiones de las mujeres madres de familia y parecieran ser el principal motivo para desarrollar iniciativas que les permitan generar ingresos -ya sea para contribuir al presupuesto familiar 0 suplir completamente las necesidades económicas- sin descuidar las tareas domésticas y el cuidado de los hijos, la literatura sugiere que existen otros factores más allá de esta relación.

Ya que como se plantea en esta revisión documental, la literatura refiere que el factor más sobresaliente los emprendimientos realizados por mujeres que son madres de familia, radica en que prevalece la motivación por hacer algo diferente, que logre dar solución a las múltiples problemáticas de los productos que ya se encuentran en el mercado, ya que los emprendimientos a menudo surgen de la experiencia de las propias madres de familia y en su desarrollo, conforman redes de cooperación donde se integran otras mujeres, madres de familia y dan cabida a grupos vulnerables cuya contribución final es social. 
En conclusión, la decisión de las mujeres madres de familia de llevar a cabo un emprendimiento, es una oportunidad para conciliar la relación familia-trabajo, además de promover el desarrollo de la autoestima, el reconocimiento, la búsqueda de independencia y autonomía a través de sus habilidades y talentos enfatizando su importancia en la bú squeda por la igualdad de género y sus aportaciones no sólo a la economía, sino su trascendencia a futuras generaciones, pues formar a una mujer es formar a la nación entera.

\section{Referencias}

Alcañiz, M. (2015). Género con clase: la conciliación desigual de la vida laboral y familiar. Revista Española de Sociología(23), 29-55. Obtenido de https://recyt.fecyt.es/index.php/res/article/view/65 362

Andersson, J. (2018). How does motherhood affect selfemployment performance. Small Business Economics, 29-54.

Brunet, I., \& Pizzi, A. (2017). Discursos de género de las mujeres emprendedoras por oportunidad. El caso español. Revista Austral de Ciencias Sociales(32), 167-184.

Brunet, I., \& Santamaría, C. (2016). La economía feminista y la división sexual del trabajo. Culturales, Época II, IV(1), 61-86. Obtenido de DialnetLaEconomiaFeministaYLaDivisionSexualDelTrab ajo-5614813\%20(1).pdf

Bueno, C. (2009). El rol de las mujeres en los cambios y continuidades de la economía informal. Argumentos, 22(60), 211-239. Obtenido de https://www.redalyc.org/pdf/595/59512089011.pd $\mathrm{f}$

Cambridge University Press. (2020). Cambridge Dictionary. Cambridge, United Kingdom. Obtenido de https://dictionary.cambridge.org/es/diccionario/ing les/mumpreneur?q=mumpreneurs

Campillo, I. (2010). Políticas de conciliación de la vida laboral y familiar en los regímenes de bienestar mediterráneos: los casos de Italia y España. Política y Sociedad, 47(1), 189-213. Obtenido de https://www.researchgate.net/publication/2772664 60_Politicas_de_conciliacion_de_la_vida_laboral _y_familiar_en_los_regimenes_de_bienestar_med iterraneos_los_casos_de_Italia_y_Espana

Collins English Dictionary. (2020). Collins English Dictionary. Glasgow: Harper Collins. Obtenido de https://www.collinsdictionary.com/es/diccionario/i ngles/mumpreneur

COPRED. (2015). Discriminación laboral hacia la mujer en la Ciudad de México. Despido por embarazo. México: COPRED. Obtenido de http://data.copred.cdmx.gob.mx/wp-
content/uploads/2015/08/Informe-

Discriminaci\%C3\%B3n-Laboral-hacia-la-MujerDespido-por-Embarazo.pdf

Di Battista, E. (2017). Mompreneurs. Una Scelta di vita. Obtenido de http://www.uncomag.com/wpcontent/uploads/2017/02/Uncomag-MompreneursDi-Battista-ebook.pdf

Fernández, A. (14 de octubre de 2019). Mujeres en el mundo laboral. Obtenido de La Vanguardia: https://www.lavanguardia.com/vida/juniorreport/20180129/44376132844/mujeres-mundolaboral.html

Gamboa, C., \& Valdés, S. (mayo de 2008). Derechos de la maternidad. Estudio teórico conceptual, de Iniciativas presentadas, y de Derecho Comparado. Obtenido de Centro de Documentación, Información y Análisis de la Cámara de Diputados. LX Legislatura: http://www.diputados.gob.mx/sedia/sia/spi/SPIISS-12-08.pdf

Genin, É., Michaudville, M. E., \& Cisneros, L. (2017). ¿Por qué dejar de ser empleada para convertirse en emprendedora o profesionista independiente? Ciencias Administrativas. Teoría y Prxis, 13(1), 205-223.

Gómez Bueno, C. (2001). Mujeres y trabajo: principales ejes de análisis. Papers, Revista de Sociología, 63, 123-140. doi:http://dx.doi.org/10.5565/rev/papers/v63n0.12 10

Larraitz, A., Chaves, M. J., Carcedo, A., \& Sánchez, A. (2019). La brecha salarial entre hombres y mujeres en América Latina. En el camino hacia la igualdad salarial. . Lima: Organización Intenacional del Trabajo. Obtenido de https://www.ilo.org/wcmsp5/groups/public/--americas/---rolima/documents/publication/wcms_697670.pdf

Nel, P., Maritz, A., \& Thongprovati, O. (2010). Motherhood and Entrepreneurship: The mumpreneur phenomenon. International Journal of Organizational Innovation, 6-34.

OIT. (2010). La maternidad en el trabajo. (Segunda ed.). Ginebra: Oficina Internacional del Trabajo. Obtenido de https://www.ilo.org/wcmsp5/groups/public/--dgreports/---dcomm/--publ/documents/publication/wcms_142159.pdf

OIT. (2016). Maternidad, trabajo e ingresos: ¿cuáles son los costos y los beneficios y cómo se distribuyen? Obtenido de https://www.ilo.org/wcmsp5/groups/public/--americas/---ro-lima/---srolima/documents/publication/wcms_493913.pdf

OXFAM México. (2020). El piso pegajoso, el techo de cristal y las nuevas revoluciones necesarias. Obtenido de https://www.oxfammexico.org/historias/el-piso- 
pegajoso-el-techo-de-cristal-y-las-nuevas-

revoluciones-necesarias

Richomme-Huet, K., \& Vial, V. (2014). Business Lessons from "Mompreneurs" Network. Global Business and Organizational Excellence, 33(4), 18-27. Obtenido de doi.org/10.1002/joe.21550

Rodríguez, M. C., \& Fernández, C. (2010). Empleo y maternidad: el discurso femenino sobre las dificultades para conciliar familia y trabajo. Cuadernos de relaciones Laborales, 28(2), 257275. Obtenido de https://www.forofamilia.org/documentos/FAMILI A\%20-

$\% 20$ Empleo\%20y\%20maternidad.El\%20discurso $\% 20$ femenino\%20sobre\%20las\%20dificultades $\% 2$ 0para\%20conciliar\%20familia\%20y\%20trabajo..p df

Smithsonian Magazine. (10 de mayo de 2019). Meet Marion Donovan, the Mother Who Invented a Precursor to the Disposable Diaper. Obtenido de https://www.smithsonianmag.com/innovation/mee t-marion-donovan-mother-who-inventedprecursor-disposable-diaper-180972118/

Todaro, R., L., G., \& Abramo, L. (2002). Desempeño laboral de hombres y mujeres: opinan los empresarios. Cadernos Pagu versión Online, 17 18. Obtenido de doi.org/10.1590/S010483332002000100008

Vargas, I. (20 de septiembre de 2011). Mamá: aprende a ser una "mompreneur". Obtenido de Expansión: https://expansion.mx/emprendedores/2011/09/19/ mama-aprende-a-ser-una-mompreneur

Vela, E. (2017). La discriminación en el empleo en México. México: CONAPRED. Obtenido de https://www.conapred.org.mx/documentos_cedoc/ completoDiscriminacion08122017.pdf

Villaécija, R. (4 de 4 de 2011). Las "mompreneur", una fuerza laboral en Francia. Obtenido de Expansión: https://www.expansion.com/201 1/04/04/empleo/m ercado-laboral/1301908952.html 